\title{
Spiral density-wave structure parameters in the solar neighbourhood derived from longitudinal velocities of Gaia EDR3 OB stars: 3D approach
}

\author{
Evgeny Griv, ${ }^{1 \star}$ Michael Gedalin, ${ }^{1}$ Przemek Mróz, ${ }^{2,3}$ De-Jian Liu ${ }^{4,5}$ and Ing-Guey Jiang ${ }^{๑ 6,7}$ \\ ${ }^{1}$ Department of Physics, Ben-Gurion University of the Negev, PO Box 653, Beer-Sheva 8410501, Israel \\ ${ }^{2}$ Division of Physics, Mathematics and Astronomy, California Institute of Technology, Pasadena, CA 91125, USA \\ ${ }^{3}$ Astronomical Observatory, University of Warsaw, Al. Ujazdowskie 4, PL-00-478 Warszawa, Poland \\ ${ }^{4}$ Purple Mountain Observatory, Chinese Academy of Sciences, Nanjing 210023, China \\ ${ }^{5}$ College of Science, China Three Gorges University, Yichang Shi 443002, China \\ ${ }^{6}$ Department of Physics and Institute of Astronomy, National Tsing-Hua University, Hsinchu 30013, Taiwan \\ ${ }^{7}$ Center for Informatics and Computation in Astronomy, National Tsing-Hua University, Hsinchu 30013, Taiwan
}

Accepted 2021 October 8. Received 2021 October 8; in original form 2021 June 22

\begin{abstract}
Distances and transverse along the Galactic longitude velocities $v_{l}$ of 2640 Gaia EDR3 O-B2 stars of Xu et al. within $2.5 \mathrm{kpc}$ from the Sun and $250 \mathrm{pc}$ from the Galactic plane with relative distance and velocity accuracies of $<10$ and $<50$ per cent are selected. Under the assumption of spiral density waves, both Galactic differential rotation parameters and parameters of the wave structure in this solar neighbourhood are derived from observed $v_{l}$. In contrast to all preceding studies, we take into account the effect of small but finite thickness of the disc of the Galaxy on even parity ('sausage') gravity perturbations of the kind investigated by Lin and Shu. As previously predicted by the modified theory of 3D density waves, two scales of periodic rarefaction-compression irregularity of the $v_{l}$ velocity field with the radial $\lambda_{0} \approx 1.5 \mathrm{kpc}$ and vertical $\xi_{0} \approx 1.0 \mathrm{kpc}$ wavelengths in the form of a spiral wave propagating in the disc are revealed. The Gaia's DR2 line-of-sight and EDR3 longitudinal velocities analyses performed in the last papers of a series exhibit consistent findings, thus providing a further evidence to support the Lin-Shu density-wave proposal.
\end{abstract}

Key words: Galaxy: kinematics and dynamics-Galaxy: structure-galaxies: spiral.

\section{INTRODUCTION}

Because of the long-range nature of the gravitational forces between entities, self-gravitating systems - planetary rings, protolunar and protoplanetary discs, the solar nebula and galaxies - exhibit collective oscillation modes in which the matter moves coherently in large regions. What is beyond controversy is that spiral arms observed in rotationally supported galaxies are collective motions of a partially self-gravitating galactic disc. Spiral structures in disc galaxies composed of stars and gas are considered as an oscillating density-wave pattern (Lin \& Shu 1964, 1966; Lin, Yuan \& Shu 1969; Shu 1970). First the theory was constructed where a wave propagating in a razor-thin disc is supposed to be quasi-stationary. The major developments in the original Lin-Shu theory of steady density waves have been nicely summarized by Binney \& Tremaine (2008). ${ }^{1}$ Then it has been shown that discrete, weakly unstable gravitational modes can be obtained from an asymptotic analysis (Lin \& Lau 1979; Bertin 1980; Bertin et al. 1989). Indeed, a differentially rotating and spatially inhomogeneous self-gravitating astrophysical disc must always be unstable, and correspondingly wave structures

\footnotetext{
*E-mail: griv@bgu.ac.il

${ }^{1}$ Using accurately determined Gaia EDR3 parallaxes and ages of 3794 open clusters, Hao et al. (2021) have recently shown that a progressive character of the nearby spiral arms is in reasonable agreement with the predictions of the original Lin-Shu-type density-wave theory.
}

developed in the disc must be time-dependent (e.g. Griv et al. 2000; Binney \& Tremaine 2008). The wave propagation is a process of rotation around the disc centre with a constant pattern speed $\Omega_{\mathrm{p}}=$ $\mathfrak{R} \omega_{k} / m$ where $\omega_{k}$ is some complex frequency of excited oscillations, suffixes $\boldsymbol{k}$ denote the kth Fourier component and $m$ is the azimuthal mode number (= number of spiral arms for a given harmonic), despite the differential rotation of the system, and of growth in amplitude and wavelength. The wave structure may be excited by real instabilities of small-amplitude gravity perturbations, for instance, those produced by a spontaneous disturbance, i.e. emerged from noise in the initial density and gravitational potential distribution (Lin \& Lau 1979; Griv, Gedalin \& Yuan 2006; Griv \& Wang 2014; Sellwood \& Carlberg 2019; Sellwood 2020), a central bar (Feldman \& Lin 1973; Korchagin \& Marochnik 1975; RomeroGómez et al. 2007; Athanassoula et al. 2010; Cheung et al. 2013) or a companion system (Goldreich \& Tremaine 1979, 1980; BlandHawthorn et al. 2019). The growth rate of the instability which causes initial perturbations to grow to observable amplitudes has a maximum at the radial wavelength $\lambda_{\text {crit }}$. As a result, of all the harmonics of the initial perturbation, the perturbation with $\lambda_{\text {crit }}$ and the associated number of spiral arms $m_{\text {crit }}$ will be formed in a time. Like sound waves in gases, a Lin-Shu-type density wave is associated with compression and decompression in the direction of travel, and thus these waves leave the equatorial plane of a galaxy flat (Fig. 1b). In such oscillations, galactic matter is continuously moving in and out of the arms. 


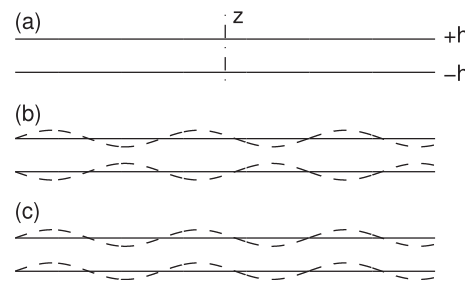

Figure 1. Drawing of perturbations of a 3D disc (cf. Griv \& Gedalin 2012, fig. 1 therein). In (a), a sector of the disc is shown edge-on. The disc rotation is taken oriented along the vertical $z$-axis. In (b) and (c), an even parity ('sausage') Lin-Shu-type and an odd ('kink') bending-type disturbances are depicted (the dashed lines), respectively.

Interestingly, the vertical bulk velocities over a radial distance range of 5-15 kpc from the Galactic Centre (GC) and up to $3 \mathrm{kpc}$ away from the Galactic plane is a mixture of breathing and bending modes, according to the LAMOST data (Ding et al. 2021), and the recent close-by passage of the Sgr dwarf galaxy cannot provoke the observed breathing oscillations in the vertical disc (Bennett \& Bovy 2021). ${ }^{2}$

In a linear approximation we are really examining, a perturbation is considered to be a combination of separate oscillation modes and the concurrence of several independent waves is possible (Lin 1971; Bertin et al. 1977; Lin \& Lau 1979). Since the components of the perturbation with different $m$ are separated, one can choose one of the Fourier harmonics at a time. Generally, $\Omega_{\mathrm{p}}$ does not depend on $m$ and therefore each $m$-component of a spiral perturbation in a system will rotate with the same angular velocity, while the $m=0$ waves in the form of concentric rings propagate away from or towards the GC (Griv et al. 2006).

As such, regular enhancements in density are causally linked to systematic variations in velocity (Lin et al. 1969; Yuan 1969; Lin, Yuan \& Roberts 1978). Hence, it is correct to investigate and explain measured velocities in terms of a density wave. Specifically, the Lindblad-Oort idea of a rotation of the Milky Way Galaxy around the GC proposes that for any type of object there is at each point in the plane a mean circular motion. A rigorously circular model is adopted, i.e. the unperturbed disc of the system is assumed to have no motion except a circular rotation and different populations of Galactic objects have different average circular velocities (e.g. Mihalas \& Binney 1998). Small non-axisymmetric perturbations of the background axisymmetric potential induce non-circular variations of stellar and gas velocities proportional to $\widetilde{v}_{r} \cos \phi, \widetilde{v}_{\varphi} \sin \phi$, and $\widetilde{v}_{z} \sin \phi$, and we are looking for these average streaming motions in our study. Here, $\widetilde{v}_{r}, \widetilde{v}_{\varphi}$, and $\widetilde{v}_{z}$ are the amplitudes of the radial, tangential, and vertical wave motions that depend weakly on the Galactocentric and vertical distances $r$ and $z$, and $\phi$ is the rapidly varying phase of the wave of the different modes of oscillations (see Section 2 below). In the modified Lin-Shu theory as formulated by Griv \& Gedalin (2012), both non-zero planar and vertical systematic velocities are attributed to the influence of the self-excited 3D density waves without the involvement of any external forces, such as that coming from companion galaxies, clouds of dark matter or massive globular clusters, crossing the Galactic disc.

Thus, the theory suggests some perturbed radial and transverse motions of stars and gas distributed in the form of a non-axisymmetric

\footnotetext{
${ }^{2}$ We do not support the use of the term 'breathing' to describe 'sausage' wave motions. The term 'breathing' is changed correspondingly to 'Lin-Shu-type density-wave' or 'sound-wave' throughout the paper.
}

flow field, even in the absence of any external perturbation (or a bar), which is a correction to the equilibrium circular motion and these streaming motions can be used as an important test of the theory (Yuan 1969; Burton 1971; Rohlfs 1977; Marochnik \& Suchkov 1981). Actually, the presence of such structure in a velocity field provides direct support for the theory. In this connection, the disc of the Galaxy is almost certainly not an axisymmetric system at equilibrium (Bovy 2017; Antoja et al. 2018; Gaia Collaboration 2018); some oscillations viewed in the rotation curve of the system under study could be linked to the physical location of the spiral arms (Lin et al. 1978; McGaugh 2016, 2019; Tarricq et al. 2021). The resulting non-circular motions in the plane are of the order of $10 \mathrm{~km} \mathrm{~s}^{-1}$, but 'we can identify two regions in the Galaxy with significantly larger peculiar motions' (Reid et al. 2019, p. 8).

First Yuan (1969) and then Burton (1971), Crézé \& Mennessier (1973), Byl \& Ovenden (1978), Mishurov, Pavlovskaya \& Suchkov (1979), Grivnev (1981), Mishurov et al. (1997), Siebert et al. (2012), and others (e.g. Fernández, Figueras \& Torra 2001) have analysed the velocities of $\mathrm{HI}$ gas, stars, H II regions and open clusters to find evidence of streaming motions of matter in our part of the Galactic plane induced by the perturbing gravitational field of the waves by using a $2 \mathrm{D}$ approach. In the first part of the work (Griv, Gedalin \& Jiang 2021), we have examined distances and line-of-sight velocities for 964 OB stars identified in the Gaia astrometry mission DR2 by Xu et al. (2018). The data were used to find small systematic departures of velocities from the mean circular motion for the stars near the Sun due to the $2 \mathrm{D}$ density waves, and revised parameters of oscillations were successfully obtained. In this work, we continue the study by taking the newly derived Gaia EDR3 distances and transverse velocities for O and early B-type stars from Xu et al. (2021). A robust statistical method is applied to investigate the observational data, and the results are compared to determinations already inferred from the $\mathrm{Xu}$ et al. (2018) ones. Consequently, various results can be compared and any consistency evaluated. Moreover, this paper is the first attempt to consider a vertical, normal to the plane motion of stars in the field of 3D gravity oscillations.

To emphasize it again, our achievement in this paper is the consideration of non-axisymmetric density waves developing in the 3D disc of the Galaxy. Young objects of the disc have nearly circular orbits, rotating around the GC with a velocity given by the rotation curve. Young stars and gas in rotationally supported galaxies are concentrated within the dynamically cold disc with typical thickness of several tens parsecs. In the framework of the theory, in such extremely thin systems the effect of small but finite thickness on 'sausage' perturbations of the kind studied by Lin and Shu is expected to be rather small in comparison with other effects. The corrugated modes of odd symmetry with respect to the equatorial $z=0$ plane (Fig. 1c), which makes the disc bend in the same way as the plane of a vibrating membrane does and do not cause density enhancements (e.g. Bland-Hawthorn et al. 2019; Hao et al. 2021), deserve an additional study.

As was pointed out to the authors by the referee of the paper, the work by Bobylev \& Bajkova (2015) shares some background with ours. By using a different, i.e. periodogram analysis, Bobylev \& Bajkova have examined trigonometric parallaxes measured by means of VLBI (e.g. Reid et al. 2014) and spatial velocities of 107 masers. These masers are related to very young $\lesssim 10^{7}$ yr old objects located in active star-forming regions. A wave in vertical velocity versus distance from the Galactic rotation axis, that is to say, a radial wavelength, was detected. Unlike Bobylev \& Bajkova, however, we determine here both radial wavelength and vertical wavelengths. Leaving aside the criticism of the value of a wavelength obtained by 

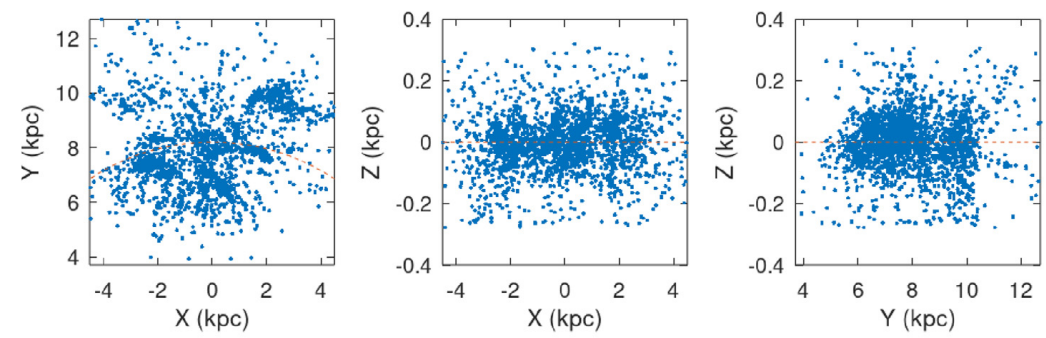

Figure 2. Space locations of all $9750 \mathrm{OB}$ stars investigated. The heliocentric Cartesian $(X, Y, Z)$ coordinates are shown (the $\mathrm{GC}$ is in the negative $Y$-direction and the rotation of the disc is in the positive $X$-direction). A dashed line indicates the solar circle and the equatorial plane. The Sun is at $(0,8.15,0.015) \mathrm{kpc}$. The sample is distributed not uniformly, and one can directly see the neighbouring inner Sagittarius and outer Perseus spirals, and the local Orion spiral between them in the plane $X Y$. The distribution of stars along the arm-segments is lumpy.

Bobylev \& Bajkova, notice also that their definition - 'the amplitudes of the radial and tangential components of the perturbed velocity which, for convenience, are always considered positive' (Bobylev \& Bajkova 2015, p. 51) - undoubtedly contradicts the predictions of the Lin and Shu density-wave theory (e.g. Mishurov et al. 1979, p. 149). The latter did not allow them as well as Bobylev, Bajkova \& Stepanishchev (2008), Bobylev \& Bajkova (2010, 2014), and Bobylev et al. (2021) to define correctly the radial phase of the Sun in the wave (see Section 2 for an explanation).

The organization of the paper is as follows. In Section 2, the details of the method used to recover the properties of the 3D density waves are outlined. Section 3 describes the Xu et al. (2021) catalogue of OB stars, and the selection of objects that was performed. Results of the model calculations are presented in Section 4. We debate the main results and draw concluding remarks in Section 5.

\section{COMPUTATIONAL METHOD}

Based on Lin et al. (1969), Yuan (1969), Shu (1970), and Griv \& Gedalin (2012), in the Galaxy total potential of the disc is written in the simple form:

$\Phi_{\text {total }}(r, \varphi, z, t)=\Phi_{\text {basic }}(r, z)+\widetilde{\Phi}(r, z) \cos \phi \cos (q z)$,

where $\Phi_{\text {basic }}(r, z)$ is a background axisymmetric potential, $\widetilde{\Phi}$ is the amplitude of the perturbing non-axisymmetric potential, $\left|\widetilde{\Phi} / \Phi_{\text {basic }}\right| \ll 1, \phi=\phi_{0}-m\left[\Omega_{\mathrm{p}} t+\varphi-(1 / \tan p) \ln \left(r / r_{0}\right)\right]$ is the phase of oscillations, $\phi_{0}$ is the phase of the wave at the Sun's location of the different modes, $t$ is a time, the angle $\varphi$ is measured clockwise in the direction of the Galactic rotation from the radius passing through the location of the Sun (Fig. 2, left-hand panel), $p$ is the pitch angle and $q>0$ is the vertical wavenumber. By definition, both $\Phi_{\text {basic }}$ and $\widetilde{\Phi}$ are negative. In the lowest order of approximation of the theory, potential minima (maxima) correspond with surface-density maxima (minima). The centre of the spiral arm corresponds to phase $\phi=0$, $\pm 2 \pi, \ldots$ and the interarm region corresponds to $\phi= \pm \pi, \pm 3 \pi, \ldots$. The system is axisymmetric in the mean.

The motion of objects is presented respectively in the form of a mean circular $V_{\text {circ }}=r \Omega$ and additional radial, tangential and vertical perturbing motions, where $\Omega$ is the angular velocity of the mean motion at the star's distance $r$ from the GC for the type of object considered. The components of the varying streaming velocity of a star are given by

$v_{r}=\widetilde{v}_{r} \cos \phi \cos (q z)$,

$v_{\varphi}=\widetilde{v}_{\varphi} \sin \phi \cos (q z)$ $v_{z}=\widetilde{v}_{z} \sin \phi \sin (q z)$

where the amplitudes $\widetilde{v}_{r}, \widetilde{v}_{\varphi}, \widetilde{v}_{z}$ are to be found, $q=\sqrt{|k| / h}, k=2 \pi / \lambda$ is the radial wavenumber, $\lambda=2 \pi r \tan p / m$ is the radial wavelength for given $p$ and $m$ (the radial spacing between adjacent arms), $h$ is the equilibrium thickness of the disc and $\Omega(r, z)$ is a smoothly varying function of $r$ and $z$ (Crézé \& Mennessier 1973; Byl \& Ovenden 1978; Mishurov et al. 1979; Griv \& Gedalin 2012). Also, $|k| h \lesssim 1$, and thus the vertical wavelength $\xi=2 \pi / q$ is of the order of (smaller than) the radial wavelength $\lambda$ (Griv \& Gedalin 2012). The radial velocity $v_{r}$ is directed outward the GC, $v_{\varphi}$ is directed along Galactic rotation, and $v_{z}$ is directed out of the plane. For the perturbations of planar velocities, we use the results of Lin et al. (1969), Yuan (1969), and Rohlfs (1977). For the perturbations of vertical velocity, the predictions of the modified theory of density waves are used, in which the finite thickness effects of the disc are taken into proper account. Accordingly, similar to the $v_{r}$ and $v_{\varphi}$, the $v_{z}$ velocity also shows a sound-wave behaviour, with a normal to the plane periodic rarefaction-compression pattern of even symmetry (equation 4$){ }^{3}$

In equations (1)-(4), the geometrical form of an arm is represented by a logarithmic function of pitch angle $p=\arctan (m / k r)=$ const. The radial distance between the spiral arms in the solar vicinity, or the local radial wavelength $\lambda_{0}=2 \pi r_{0}|\tan p| / m$, and the subscript ' 0 ' denotes values corresponding to the position of the Sun. The arms of the Galaxy are described by trailing spirals with $p \approx-10^{\circ}$ (Hou \& Han 2014; Nakanishi \& Sofue 2016; Reid et al. 2019; CantatGaudin et al. 2020; Hao et al. 2021). The phase $\phi_{0}$ determines the Sun's position with respect to the arms. Values of $\phi_{0}= \pm \pi n, n=0,2$, $\ldots$, correspond to the middle of the arms (potential minima/density maxima) and $n=1,3, \ldots$ correspond to the centres of interarm regions (potential maxima/density minima) in the field of the arms. The positions at the outer (inner) edges of arms are defined by $\phi_{0}$ $=-\pi / 2,3 \pi / 2,-5 \pi / 2, \ldots\left(\phi_{0}=\pi / 2,5 \pi / 2,-3 \pi / 2, \ldots\right)$. The amplitudes $\widetilde{\Phi}, \widetilde{v}_{r}, \widetilde{v}_{\varphi}, \widetilde{v}_{z}$ and the wavenumbers $k, \xi$ are slowly varying functions of $r$ and $z$ compared to $\phi$, and we consider them to be constants.

\footnotetext{
${ }^{3}$ Widrow et al. (2012), Williams et al. (2013), and Carlin et al. (2013) have already revealed coherent oscillations of $v_{z}$ versus the $z$-distance in the stellar disc in SEGUE, RAVE, and LAMOST observations, respectively. The vertical number-count density asymmetry has been also studied by Yanny \& Gardner (2013) and Bennett \& Bovy (2019). To reiterate, in our model spiral arms are considered as a possible source of the internal disturbances to the disc and create sound-wave structures in the mean planar and vertical velocities (and density!).
} 
If we consider the region $\Omega_{\mathrm{p}}<\Omega(r)\left(\Omega_{\mathrm{p}}>\Omega(r)\right)$ and $q|z|<\pi / 2$, then equations of the theory state that the central part of a spiral arm is moving towards (away from) the GC, $v_{r}<0\left(v_{r}>0\right)$, and the interarm region is moving away from (towards) it. The outer edge of an arm exhibits a perturbing azimuthal motion in the direction of Galactic rotation and the inner edge exhibits an azimuthal motion in the direction opposite to rotation. The direction of the azimuthal motion changes in the middle of the arm and interarm regions, while the direction of the radial motion changes at the inner and outer edges of spiral arms (Lin et al. 1969; Yuan 1969; Rohlfs 1977). Similar to the streaming tangential velocity, the $v_{z}$ velocity has its extreme values for $\phi= \pm \pi / 2, \pm(3 / 2) \pi, \ldots$, that is, at the outer and inner spiral arm edges, and the centres of spiral arms and interarm regions exhibit no any vertical wave motion. At the outer edge of the spiral arm the medium both above and below the plane are moving on average outwards (towards) the $z=0$ plane, while at the inner edge of the spiral arm the matter is moving towards (outwards) the equatorial plane (Griv \& Gedalin 2012). Thus, the direction of the vertical motion changes in the middle of the arms and interarm regions $(\phi=0, \pm \pi, \pm 2 \pi, \ldots)$.

Observed values of heliocentric line-of-sight, longitudinal and latitudinal components of a star's velocity $v_{\text {los }}, v_{l}, v_{b}$ corrected for solar motion towards the apex are

$$
\begin{aligned}
v_{\mathrm{los}}= & \cos b\left[r_{0}\left(r-r_{0}\right) \dot{\Omega}_{0} \sin l\right. \\
& -\widetilde{v}_{r} \cos \phi \cos (q z) \cos (l+\varphi)+\widetilde{v}_{\varphi} \sin \phi \cos (q z) \sin (l+\varphi) \\
& \left.+u_{0} \cos l-v_{0} \sin l\right]+\left[\widetilde{v}_{z} \sin \phi \sin (q z)-w_{0}\right] \sin b,
\end{aligned}
$$

$v_{l}=\left(r-r_{0}\right)\left(r_{0} \cos l-d \cos b\right) \dot{\Omega}_{0}$

$$
+\widetilde{v}_{r} \cos \phi \cos (q z) \sin (l+\varphi)+\widetilde{v}_{\varphi} \sin \phi \cos (q z) \cos (l+\varphi)
$$$$
-u_{0} \sin l-v_{0} \cos l-\Omega_{0} d \cos b \text {, }
$$

$$
\begin{aligned}
v_{b}= & -\sin b\left[r_{0}\left(r-r_{0}\right) \dot{\Omega}_{0} \sin l\right. \\
& -\widetilde{v}_{r} \cos \phi \cos (q z) \cos (l+\varphi)+\widetilde{v}_{\varphi} \sin \phi \cos (q z) \sin (l+\varphi) \\
& \left.+u_{0} \cos l-v_{0} \sin l\right]+\left[\widetilde{v}_{z} \sin \phi \sin (q z)-w_{0}\right] \cos b,
\end{aligned}
$$

where $l$ and $b$ are the Galactic coordinates, $v_{l}$ and $v_{b}$ are the measured transverse along the longitude and along the latitude velocities, $\Omega_{0}\left(r_{0}\right)$ is the angular velocity of the mean motion at $r_{0}$ and the constants $u_{0}, v_{0}, w_{0}$ are the components of solar peculiar motion relative to the mean linear speed of rotation $r_{0} \Omega_{0}$ at $r_{0}$ (Byl \& Ovenden 1978; Mishurov et al. 1979; Mihalas \& Binney 1998). In equations (5)-(7), the expansion of the angular velocity of rotation into a Taylor series up to the $n$th order of smallness in $d / r_{0}$ ( $d$ is the Sun's distance from the star),

$\Omega=\Omega_{0}+\left(r-r_{0}\right) \dot{\Omega}_{0}+\ldots$,

or $\left|\left(r-r_{0}\right) \dot{\Omega_{0}} / \Omega_{0}\right| \ll 1$ is used to give the motions of stars in a straightforward way, where a dot denotes a derivative $\mathrm{d} / \mathrm{d} r$ along $r$. Considering objects of the thin disc, all derivatives of $\Omega(r, z)$ with respect to the vertical coordinate $z$ are omitted.

Equations (5)-(7) are not exact, in the sense that they involve approximations. In the disc near the Sun, $\Omega_{0} \approx 30 \mathrm{~km} \mathrm{~s}^{-1} \mathrm{kpc}^{-1}$ and $\left|\dot{\Omega}_{0}\right| \approx 3 \mathrm{~km} \mathrm{~s}^{-1} \mathrm{kpc}^{-2}$ (Bovy 2017; Schönrich \& Dehnen 2018; Mróz et al. 2019; VERA Collaboration 2020). Including only the first derivative of $\Omega_{0}$ in the expansion (8), we therefore limit the data range to about $d \lesssim 3 \mathrm{kpc}$. Thus, doing in equations (5)-(7) an expansion of the angular velocity term, our calculation is now applied only in this solar vicinity; considerable errors may occur if $d$ is large. Both $\left|\widetilde{v}_{r}\right|,\left|\widetilde{v}_{\varphi}\right|,\left|\widetilde{v}_{z}\right|$ and $\left|u_{0}\right|,\left|v_{0}\right|,\left|w_{0}\right|$ are small compared with $r_{0} \Omega_{0}$. The radius $r$ is

$r^{2}=\left(r_{0}-d \cos b \cos l\right)^{2}+d^{2} \cos ^{2} b \sin ^{2} l+\left(d \sin b+z_{0}\right)^{2}$,

where $z_{0}$ denotes the Sun's distance from the plane.

So, the terms $\propto \Omega_{0}, \dot{\Omega}_{0}$ and $u_{0}, v_{0}, w_{0}$ describe the mean rotation of the system and the peculiar Sun's motion. The diversion of the motion of objects from the circular motion due to a wave is characterized by terms $\propto \widetilde{v}_{r} \cos \phi \cos (q z), \widetilde{v}_{\varphi} \sin \phi \cos (q z)$, and $\widetilde{v}_{z} \sin \phi \sin (q z)$. The method does not afford a decisive estimate of the pitch angle $p$ and the number of spiral arms $m$ but only of the radial $\lambda_{0}$ and vertical $\xi_{0}$ wavelengths for given $\phi_{0},(p, m)$ and $h$. Especially notice that equations (5)-(7) describe sound-wave behaviour of the matter both in the plane and in the perpendicular to the plane direction.

For stars in the nearby disc, the line-of-sight velocities (measured by the Doppler effect in stellar spectra) and the latitudinal velocities (measured from the proper motion) show a 'double sine wave' as a function of $l, v_{\text {los }}$ and $v_{b} \propto \sin (2 l)$, while the longitudinal velocities (measured from the proper motion) show a 'double cosine wave', $v_{l}$ $\propto \cos (2 l)$ (Mihalas \& Binney 1998).

The young Galactic disc is thin because the vertical motions of stars, which are responsible for the thickness of the stellar disc, are low compared with $V_{\text {circ }}$. Young stars, in particular massive OB stars, are concentrated within the dynamically cold thin disc with typical $h$ of several tens parsecs only (e.g. Skowron et al. 2019). In the equations above, considering the flat-component objects, the terms $\propto \sin b$ and $\sin (q z)$ are therefore relatively small compared with those ones $\propto \cos b$ and $\cos (q z)$.

In equations (5)-(7), the quantities $\widetilde{v}_{r}, \widetilde{v}_{\varphi}, \widetilde{v}_{z}$ are periodic with to $\phi_{0}$ with period $2 \pi$, while the quantity $S$ is periodic with to $\phi_{0}$ with period $\pi$. Correspondingly, from the $S\left(\phi_{0}\right)$ relation alone we cannot conclude in what region $\phi_{0}=0^{\circ}-180^{\circ}$ or $\phi_{0}=180^{\circ}-360^{\circ}$ the Sun is actually located (Mishurov et al. 1979). In sharp contrast to Bobylev et al. (2008, 2021) and Bobylev \& Bajkova (2010, 2014, $2015)$, to resolve the uncertainty, for a trailing pattern $(k<0)$, one has to choose for the phase $\phi_{0}$ the values that satisfy the strong condition:

$\widetilde{v}_{\varphi}<0$

(Mishurov et al. 1997, p. 777). ${ }^{4}$

The velocity field is now described approximately by the rotation parameters $\left(\Omega_{0}, \dot{\Omega}_{0}\right)$, the peculiar solar motions $\left(u_{0}, v_{0}, w_{0}\right)$ relative to the anticentre, in the direction of rotation and in the direction of the North Galactic pole and the amplitudes $\left(\widetilde{v}_{r}, \widetilde{v}_{\varphi}, \widetilde{v}_{z}\right)$ of the non-circular streaming velocity in the radial, tangential, and vertical directions. The quantities $\lambda_{0}$ and $\xi_{0}$ describe the radial and vertical scales of irregularity of the velocity field in the neighbourhood of the Sun, and the quantity $\phi_{0}$ defines the Sun's position with respect to the arms. Since only the transverse velocities are available for Xu et al.'s (2021) objects, only equations (6) and (7) can be used to determine the parameters of the wave pattern, differential rotation, and peculiar motion of the Sun. We can thus find the values of $\Omega_{0}$, $\dot{\Omega}_{0}, u_{0}, v_{0}, w_{0}, \widetilde{v}_{r}, \widetilde{v}_{\varphi}, \widetilde{v}_{z}, \lambda_{0}$ (and $\xi_{0} \equiv \sqrt{2 \pi \lambda_{0} h}$ ) for given sets of $\phi_{0}$ and $(p, m)$ from observed velocities $v_{l}$ and $v_{b}$. The uncertainties

\footnotetext{
${ }^{4}$ Bobylev et al. $(2008,2021)$ and Bobylev \& Bajkova $(2010,2014,2015)$ have also estimated the amplitudes of the radial and tangential components of the perturbed velocity in a rough manner by using the $\widetilde{v}_{r}\left(\widetilde{v}_{\varphi}\right)=\sqrt{4 \mathcal{A}}$ relation, where $\mathcal{A}$ is the peak value of the power spectrum.
} 

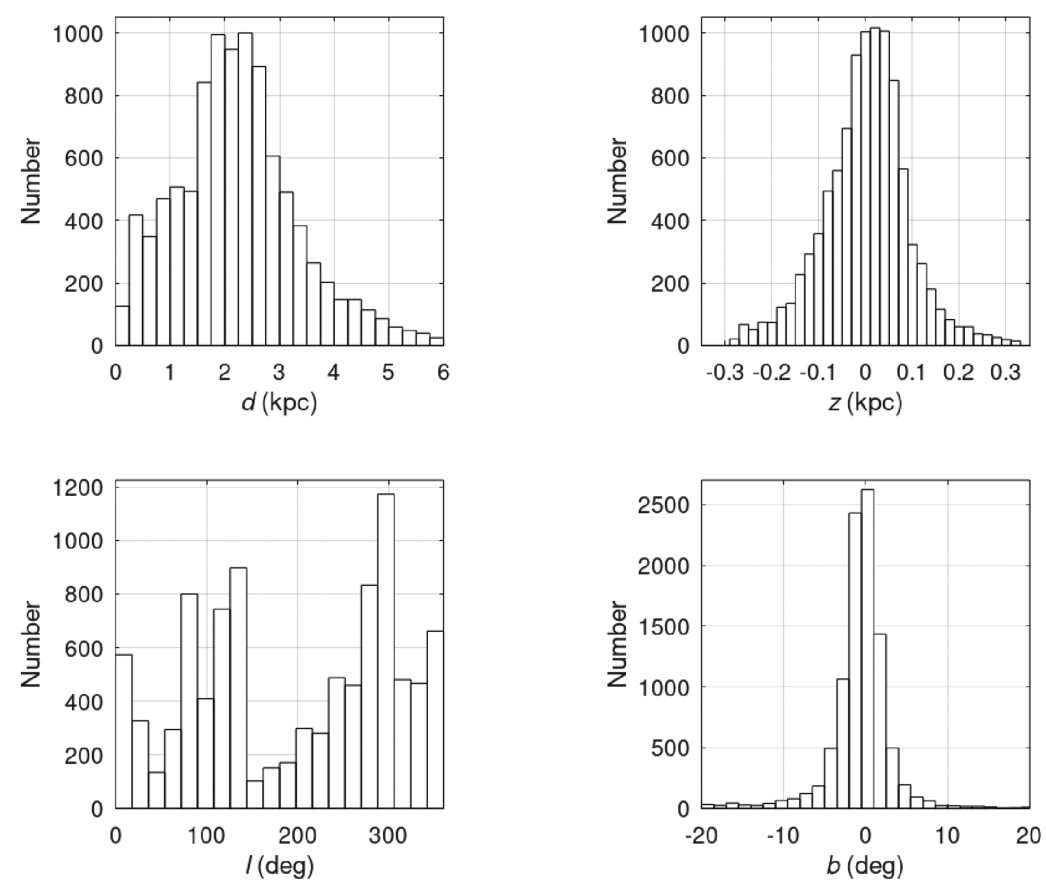

Figure 3. Distribution of OB stars with respect to $d$ (left-top panel), $z$ (right-top panel), $l$ (left-bottom panel), and $b$ (right-bottom panel).

in the parameters $\Omega_{0}, \ldots, \lambda_{0}$ are determined by means of numerical experiments (Pavlovskaya \& Suchkov 1980).

\section{STELLAR DATA}

The catalogue of stars recently published by $\mathrm{Xu}$ et al. (2021) is explored. Totally 9750 O-B2 stars located in the Galactic disc for which the trigonometric parallaxes $\varpi$ and proper motions $\mu_{\alpha} \cos b$, $\mu_{\delta}$ are available in the Gaia EDR3 were classified. The mean age of the sample is $\approx 10^{7} \mathrm{yr}$. It is the largest sample of spectroscopically confirmed massive, very young stars available to date, which was already used to trace directly the spiral pattern within $\approx 5 \mathrm{kpc}$ of the Sun. OB stars are typically metal-rich stars belonging to Population I, located in the thin disc subsystem. We convert a parallax to a distance, $d=1 / \varpi$, neglecting the possible bias of about 0.017 mas on the Gaia EDR3 parallax zero-point for the OB stars. The distances of the Sun from the GC and the plane are $r_{0} \approx 8.15 \mathrm{kpc}$ and $z_{0}$ $\approx 0.015$ kpc (Bland-Hawthorn \& Gerhard 2016; McMillan 2017; Bennett \& Bovy 2019; GRAVITY Collaboration 2019; Griv et al. 2019; Reid et al. 2019; Skowron et al. 2019; VERA Collaboration 2020).

Fig. 2 displays locations of all OB stars projected on to the $X Y$, $X Z$, and $Y Z$ planes (the $X$-axis points to the direction of the Galactic rotation, the $Y$-axis points to the anticentre, the $Z$-axis points to the North Galactic pole and the axis of the rotation is taken oriented along the $0 Z$-axis). The nearby spiral structure can be derived immediately from the positional data as displayed in Fig. 2 (Xu et al. 2021). There are three periodic spiral signals in the $X Y$ plane evident to the eye in the part of the Galaxy wherein the Sun is located, namely the Sagittarius, Orion, and Perseus spiral arm segments from bottom to top (segments of global spiral arms similar to the spirals in the Andromeda galaxy?). The distribution of stars along the armsegments is not homogeneous (cf. Chen et al. 2019). Xu et al.
(2021) have pointed out that in the arms the distribution of stars is spotty, which may be a result of the irregular distribution of giant molecular clouds. In the Galaxy, very young populations - $\mathrm{O}$ and early B-type stars, young Cepheids and open clusters with ages $t_{\text {age }}$ $<5 \times 10^{7} \mathrm{yr}$, masers associated with high-mass stars, $\mathrm{H}$ II regions, giant molecular clouds and $\mathrm{H}$ I gas - are the standard tracers of spirals (Nakanishi \& Sofue 2016; Xu et al. 2018; Chen et al. 2019; Reid et al. 2019; Skowron et al. 2019; Cantat-Gaudin et al. 2020; Hao et al. 2021; Poggio et al. 2021; Tarricq et al. 2021). The models of large-scale $m=3$ and $m=4$ logarithmic spirals with $|p|=10^{\circ}-$ $15^{\circ}$ are capable to connect most arm tracers (Hou \& Han 2014). Castro-Ginard et al. (2021) have deduced values of $|p|=9^{\circ}-16^{\circ}$ for Scutum, Saggitarius, Orion, and Perseus arms using the most complete open clusters sample to date, with astrometric Gaia EDR3 updated parameters, estimated astrophysical information and radial velocities.

The distributions of stars with respect to $d, z, l$, and $b$ are then shown in Fig. 3. The sample objects fill the region $r_{0} \pm 2.5 \mathrm{kpc}$ almost uniformly (Fig. 3, left-top panel). The stars are prevalently limited within $\approx 250$ pc below and above the $z=0$ plane (Fig. 3 , righttop panel). We find an exponential scale height of about $50 \mathrm{pc}$ for the thin disc at the Sun's position, thus the effective $h \approx 100$ pc. Two maxima at $l \approx 100^{\circ}$ and $l \approx 280^{\circ}$ are seen in the distribution (Fig. 3, left-bottom panel). We tend to attribute an observed phenomenon to the fact that a large part of objects is located in the Orion arm, in which the Sun is also situated, and in the Sagittarius arm (Fig. 2, left-hand panel). For the most stars $|b|<10^{\circ}$ (Fig. 3, right-bottom panel), and therefore in equations (5) and (7) the factor $|\sin b|$ is much smaller than 1 .

Fig. 4 is a distribution of stars with respect to $v_{l}$ and $v_{b}$ and a distribution of individual random errors $\Delta v_{l}$ of $v_{l}$ and $\Delta v_{b}$ of $v_{b}$. The velocity distribution of objects is fairly close to a normal-like distribution, i.e. close to equilibrium with the means $\approx-41.5 \mathrm{~km} \mathrm{~s}^{-1}$ and $\approx-8.5 \mathrm{~km} \mathrm{~s}^{-1}$ and covers velocity ranges mainly from -200 

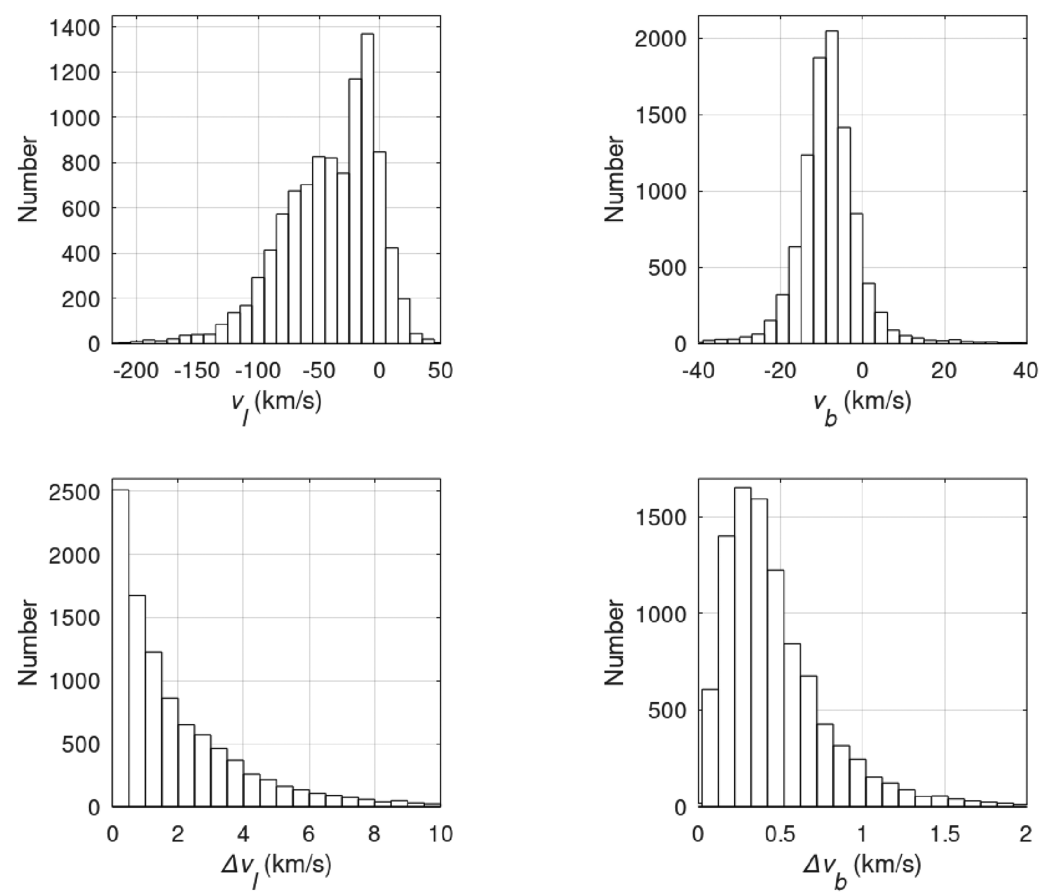

Figure 4. Velocities $v_{l}$ and $v_{b}$, and the relative uncertainties $\Delta v_{l}$ of $v_{l}$ and $\Delta v_{b}$ of $v_{b}$.
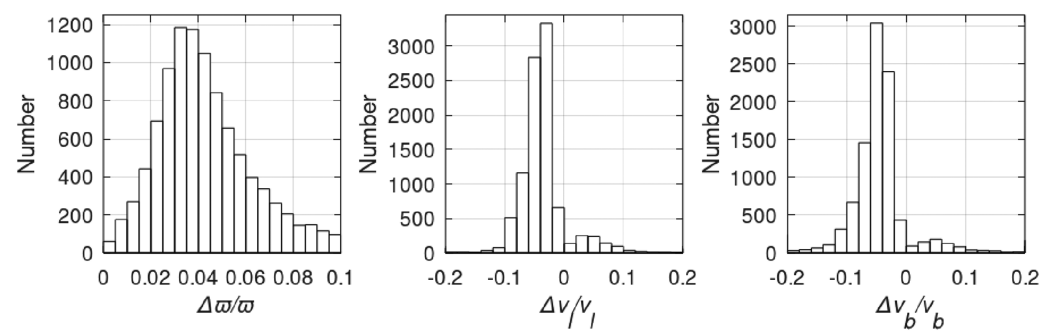

Figure 5. Distribution of stars with respect to errors $\Delta \varpi$ of $\varpi$ (left-hand panel), $\Delta v_{l}$ of $v_{l}$ (middle panel), and $\Delta v_{b}$ of $v_{b}$ (right-hand panel).

to $+50 \mathrm{~km} \mathrm{~s}^{-1}$ and -40 to $+40 \mathrm{~km} \mathrm{~s}^{-1}$, respectively. The average values of velocity errors of the sample are $\approx \pm 2.1 \mathrm{~km} \mathrm{~s}^{-1}$ for $v_{l}$ and $\approx \pm 0.5 \mathrm{~km} \mathrm{~s}^{-1}$ for $v_{b}$.

Fig. 5 shows the distribution of stars with respect to relative uncertainties of parallaxes $\Delta \varpi / \varpi$, longitudinal velocities $\Delta v_{l} / v_{l}$ and latitudinal velocities $\Delta v_{b} / v_{b}$. Almost all of the objects have a distance accuracy of better than \pm 10 per cent and a velocity accuracy of better than \pm 20 per cent.

Correlation coefficients between proper motion in right ascension RA and declination DEC and $l$ and $b$ are plotted in Fig. 6 (left-hand panel) and Fig. 6 (right-hand panel). For most objects in our sample the correlation coefficients are close to zero and therefore we can treat measurements of the proper motion as independent.

Fig. 7 gives a plot $v_{l}$ and $v_{b}$ as a function of $l$. A well-defined effect of differential rotation is clearly seen in the longitudinal velocity field for the vast majority of objects (Fig. 7, top panel). The transversemotion component $v_{b}$, however, does not show an expected 'double sine wave' as a function of $l$ (Fig. 7, bottom panel). This is very probably because the factor $|\sin b| \ll 1$ and random errors in equation (7) mask the 'double sine' effect of differential rotation. The problem is far from a solution currently but the situation may improve with more and/or better data. In the following only $v_{l}$ velocities will be examined by treating equation (6). Thus, according to this equation, only two disturbance velocities proportional to $\widetilde{v}_{r}$ and $\widetilde{v}_{\varphi}$ but not $\widetilde{v}_{z}$ will be estimated.

Based on Figs (3)-(5), we restricted ourselves to stars with their distances $d<2.5 \mathrm{kpc}$ and $|z|<250 \mathrm{pc}$, with velocities $\left|v_{l}\right|<$ $200 \mathrm{~km} \mathrm{~s}^{-1}$ and $\left|v_{b}\right|<50 \mathrm{~km} \mathrm{~s}^{-1}$, and with relative errors of parallaxes and velocities of less than 10 and 50 per cent, respectively. As simulations show, changes in the values of distances in the ranges of $2<d<4 \mathrm{kpc}$ and $50<|z|<350 \mathrm{pc}$, velocities in the ranges 100 $<\left|v_{l}\right|<300 \mathrm{~km} \mathrm{~s}^{-1}$ and $25<\left|v_{b}\right|<100 \mathrm{~km} \mathrm{~s}^{-1}$, relative errors of distances and velocities in the ranges $0.025<\Delta \varpi / \varpi<0.11$ and $0.05<\left|\Delta v_{l} / v_{l}\right|<1.0$, and inclusion of the second derivative of $\Omega_{0}$ in the expansion (8) do not affect our main conclusions. The final sample consists of $N=2640$ objects.

\section{RESULTS OF CALCULATION}

To compute the mutually independent unknowns $\Omega_{0}, \ldots, \lambda_{0}$ for given $\phi_{0}$ and $(p, m)$, we calculate the weighted least-square sum of squared residuals of measured $v_{i}^{\text {obs }}$ and modelled $v_{i}^{\text {mod }}$ (equation 6) 

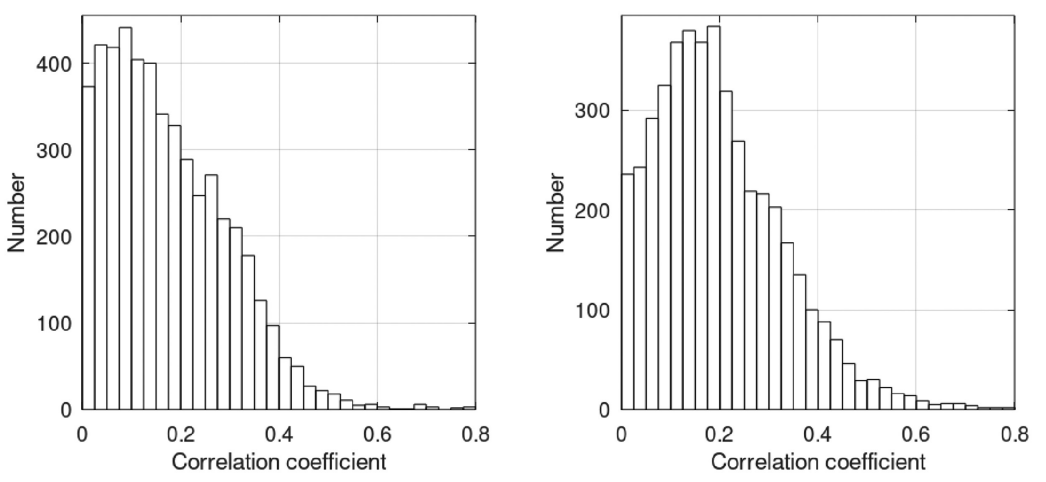

Figure 6. Correlation coefficients between proper motion in RA and DEC (left-hand panel) and $l$ and $b$ (right-hand panel).
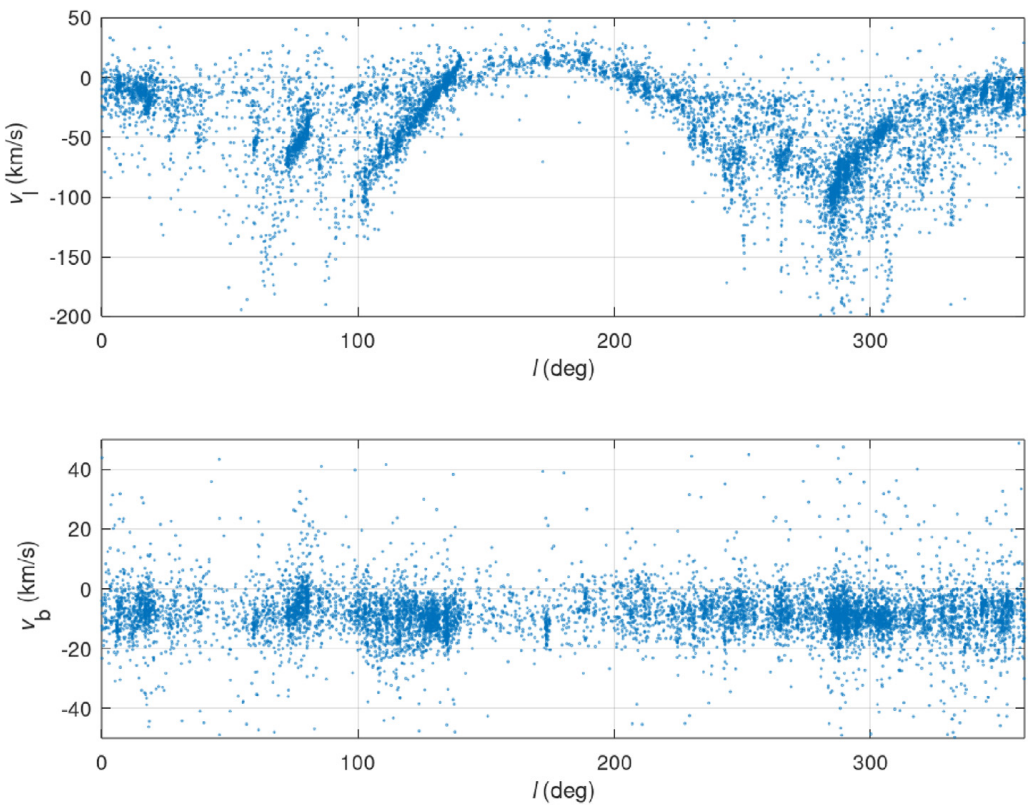

Figure 7. Transverse velocities of stars as a function of $l$ : the longitudinal velocities $v_{l}$ (top panel) and latitudinal velocities $v_{b}$ (bottom panel).

velocities as

$S=\frac{1}{N-q} \sum_{i=1}^{N}\left(\frac{v_{i}^{\mathrm{obs}}-v_{i}^{\mathrm{mod}}}{\Delta v_{i}^{\mathrm{obs}}}\right)^{2}$,

where $q=8$ is the number of parameters to be derived by the method and $\Delta v_{i}^{\text {obs }}$ is the uncertainty of $v_{i}^{\text {obs }}$. The values of $\Omega_{0}, \ldots, \lambda_{0}$ are found by fitting that minimizes $S$, using a Levenberg-Marquardt algorithm. The model depends linearly on the set of six parameters $\Omega_{0}, \dot{\Omega}_{0}, u_{0}, v_{0}, \widetilde{v}_{r}, \widetilde{v}_{\varphi}$ and non-linearly on two parameters $\phi_{0}$ and $(p$, $m$ ) (or $\lambda_{0}$ ). For each model $m$ was fixed and minimization of $S$ by the non-linear least-squares fitting was performed in $\phi_{0}$ and $\lambda_{0}$ (that is to say, in the phase of the wave $\phi_{0}$ and in the pitch angle $p$ at the solar location $r_{0}$ for a given $m$ ).

The surface of $S\left(\phi_{0}, \lambda_{0}\right)$ and cuts through the $S$ space as a function of $\phi_{0}$ and $\lambda_{0}$ constructed over longitudinal velocities of selected 2640 stars for the most interesting $m=1,2,3$, and 4 3D models are shown in Fig. 8. A highly significant global minimum of $S$ with respect to $\phi_{0}$ and $\lambda_{0}$ near $\phi_{0} \approx 0^{\circ}$ and $\lambda_{0} \approx 1.5 \mathrm{kpc}$ is viewed from our simulation for the models. We especially note the similarity between Fig. 8, on the one side, and figs 18-19 and 4 of Griv et al. (2020, 2021), respectively, on the other side. As for us, this indisputable resemblance of the $S$ surfaces obtained for the fully independent Gaia DR2 line-of-sight (Griv et al. 2020, 2021) and EDR3 longitudinal (this work) velocities is so remarkable that no doubt is felt as to the original Lin-Shu density-wave idea's truth with respect to the data. This result is also quite similar to that of Griv, Jiang \& Hou (2017) who considered coherent non-axisymmetric flows within a few kiloparsecs from the Sun of 19291 intermediate-old giant stars from the APOGEE sky survey.

Table 1 shows our results in the sample of stars for global minimum in $S$. The sum $S$, the coefficient of determination $\mathcal{R}^{2}$, the rotational parameters of the Galaxy, and the geometrical parameters of waves for the minimum in $S\left(\phi_{0}, \lambda_{0}\right)$ for the axisymmetric $m=0$ model with $\widetilde{v}_{r}, \widetilde{v}_{\varphi}$, and $\widetilde{v}_{z}=0$ and for four non-axisymmetric $m \geq 1$ models are presented. ${ }^{5}$ The main results are as follows:

${ }^{5}$ The quantity $\mathcal{R}^{2}$ is given by:

$\mathcal{R}^{2}=1-\sum_{i}\left(v_{i}^{\mathrm{obs}}-v_{i}^{\mathrm{mod}}\right)^{2} / \sum_{i}\left(v_{i}^{\mathrm{obs}}-\bar{v}_{i}^{\mathrm{obs}}\right)^{2}$,

where $v_{i}^{\text {obs }}$ are the observed velocities, $v_{i}^{\text {mod }}$ are the modelled velocities, and $\bar{v}_{i}^{\text {obs }}$ is the mean of the observed data. $\mathcal{R}^{2}$ ranges from 0 to 1 , and the best- 

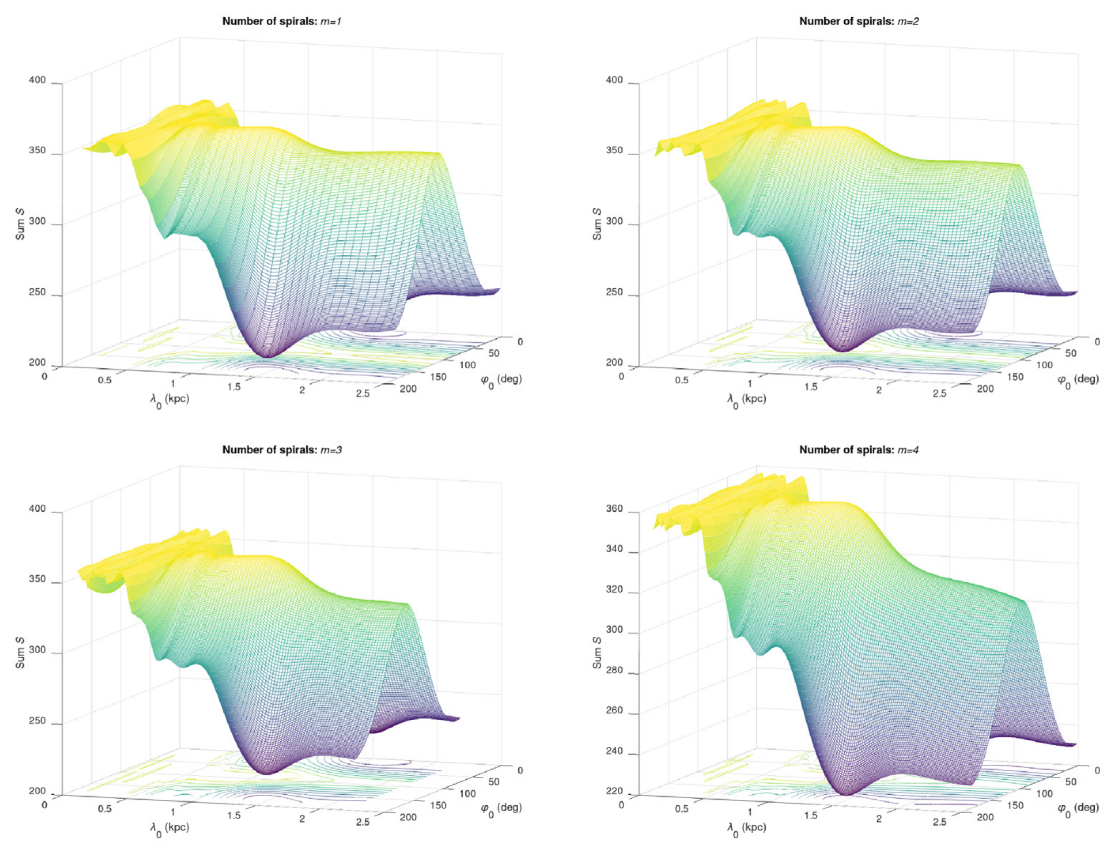

Figure 8. The surface of $S$ and cuts through the $S$ space as a function of $\phi_{0}$ and $\lambda_{0}$ for the $m=1,2,3$, and 4 models derived from $v_{l}$ velocities of selected 2640 OB stars. Because $S$ is periodic with respect to $\phi_{0}$ with period $\pi$, only the values of $S$ for the range $0^{\circ}<\phi_{0}<180^{\circ}$ are shown.

Table 1. Data on the parameters of wave structure, Galactic differential rotation, and solar peculiar motion for the $m=0-4$ models and their uncertainties derived from $v_{l}$ of selected OB stars of Xu et al. (2021). Columns 2 to 13 list the sum $S$, the coefficient of determination $\mathcal{R}^{2}$, the phase of the wave at the Sun's location $\phi_{0}$, pitch angle $p$, local radial and vertical wavelengths $\lambda_{0}$ and $\xi_{0}$, amplitudes of radial and tangential streaming velocities $\widetilde{v}_{r}$ and $\widetilde{v}_{\varphi}$, rotation parameters $\Omega_{0}$ and $\dot{\Omega}_{0}$, and, finally, radial and tangential peculiar solar velocities $u_{0}$ and $v_{0}$.

\begin{tabular}{ccccccccccccc}
\hline Model & $S$ & $\mathcal{R}^{2}$ & $\begin{array}{c}\phi_{0} \\
\left({ }^{\circ}\right)\end{array}$ & $\begin{array}{c}p \\
\left({ }^{\circ}\right)\end{array}$ & $\begin{array}{c}\lambda_{0} \\
(\mathrm{kpc})\end{array}$ & $\begin{array}{c}\xi_{0} \\
(\mathrm{kpc})\end{array}$ & $\begin{array}{c}\widetilde{v}_{r} \\
\left(\mathrm{~km} \mathrm{~s}^{-1}\right)\end{array}$ & $\begin{array}{c}\widetilde{v}_{\varphi} \\
\left(\mathrm{km} \mathrm{s}^{-1}\right)\end{array}$ & $\begin{array}{c}\Omega_{0} \\
(\mathrm{~km} /(\mathrm{skp}))\end{array}$ & $\begin{array}{c}\dot{\Omega}_{0} \\
\left(\mathrm{~km}^{2}\left(\mathrm{~s} \mathrm{kpc}^{2}\right)\right)\end{array}$ & $\begin{array}{c}u_{0} \\
\left(\mathrm{~km} \mathrm{~s}^{-1}\right)\end{array}$ & $\begin{array}{c}v_{0} \\
\left(\mathrm{~km} \mathrm{~s}^{-1}\right)\end{array}$ \\
\hline$m=0$ & +358.9 & +0.39 & & & & & & & +27.7 & -2.06 & -9.62 & +18.13 \\
& \pm 0.8 & \pm 0.01 & & & & & & & \pm 0.1 & \pm 0.02 & \pm 0.03 & \pm 0.06 \\
$m=1$ & +215.2 & +0.63 & -1.3 & -1.62 & +1.45 & +1.00 & +6.9 & -36.6 & +29.6 & -2.28 & -4.85 & +9.84 \\
& \pm 0.8 & \pm 0.02 & \pm 0.2 & \pm 0.01 & \pm 0.01 & \pm 0.01 & \pm 0.1 & \pm 0.1 & \pm 0.1 & \pm 0.02 & \pm 0.04 & \pm 0.08 \\
$m=2$ & +219.9 & +0.62 & -1.5 & -3.28 & +1.47 & +1.01 & +5.6 & -36.7 & +29.5 & -2.39 & -5.63 & +9.89 \\
& \pm 0.8 & \pm 0.02 & \pm 0.2 & \pm 0.01 & \pm 0.01 & \pm 0.01 & \pm 0.1 & \pm 0.1 & \pm 0.1 & \pm 0.02 & \pm 0.05 & \pm 0.09 \\
$m=3$ & +224.2 & +0.61 & -1.3 & -4.00 & +1.49 & +1.01 & +4.3 & -36.9 & +29.4 & -2.48 & -6.52 & +9.66 \\
& \pm 0.7 & \pm 0.02 & \pm 0.2 & \pm 0.02 & \pm 0.01 & \pm 0.01 & \pm 0.1 & \pm 0.1 & \pm 0.1 & \pm 0.02 & \pm 0.05 & \pm 0.08 \\
$m=4$ & +228.3 & +0.61 & -0.6 & -6.83 & +1.53 & +1.03 & +3.2 & -37.5 & +29.5 & -2.61 & -7.40 & +9.15 \\
& \pm 0.9 & \pm 0.02 & \pm 0.2 & \pm 0.02 & \pm 0.01 & \pm 0.01 & \pm 0.2 & \pm 0.2 & \pm 0.1 & \pm 0.02 & \pm 0.06 & \pm 0.10 \\
\hline
\end{tabular}

(i) The $S$ statistics (which are actually $\chi^{2}$ per degrees of freedom) appear to be quite high, $S \gg 1$. This may indicate that the error bars are underestimated (for example, stars show additional random motions).

(ii) An axisymmetric statistical model (i.e. spirals not included in the model) does not fit the data as well as the non-axisymmetric models: the mean sum and the coefficient of determination are $S \approx$ 360 and $\mathcal{R}^{2} \approx 0.4$ for the $m=0$ model, while $S \approx 220$ and $\mathcal{R}^{2} \approx 0.6$ for the $m \geq 1$ models. The difference in $\mathcal{R}^{2}$ of 0.2 is sufficiently large, larger than the individual error of 0.02 and larger than a $3 \sigma$ error of 0.06 for the difference. Hence, the axisymmetric model is definitely

fitting model will have $\mathcal{R}^{2}=1$. The correlation coefficient is $r_{\text {corr }}= \pm \sqrt{\mathcal{R}^{2}}$. This measures the strength of the linear relationship between $v_{i}^{\text {obs }}$ and $v_{i}^{\text {mod }}$. In the spirit of Cohen (1988), $0.1<\left|r_{\text {corr }}\right| \leq 0.3$ means a weak correlation, $0.3<\left|r_{\text {corr }}\right| \leq 0.5$ presents a moderate correlation, and $0.5<\left|r_{\text {corr }}\right| \leq 1.0$ indicates a strong correlation. not as good as any of spiral models considered. In agreement with the density-wave theory's suggestion, the existence of spiral structure in the stellar $v_{l}$ velocity field is thus founded.

(iii) A model fits the data truly well: the mean coefficient of determination is really high, $\mathcal{R}^{2} \gtrsim 0.6$, for the $m \geq 1$ models. This indicates that $\gtrsim 60$ per cent of the variation can be explained by the proposed model, so the fits are good. The correlation coefficient is $r_{\text {corr }} \approx 0.8$, which indicates a strong positive relationship. The first derivative of the angular velocity $\dot{\Omega}_{0}$ is also determined adequately. This is because the ratio $\left|\left(r-r_{0}\right) \dot{\Omega}_{0} / \Omega_{0}\right|$ is small (equation 8).

(iv) The $S$ and $\mathcal{R}^{2}$ values for fits with $m=1-4$ are practically equal. It is therefore almost impossible to understand which $m$-mode is the dominant. As a result, one scale cannot be preferred with good confidence over the other on the basis of our calculation. It could, in this case, simply mean that there is not enough detail present in the data used to reveal what mode(s) is or are the most effective. On the other hand, several low- $m$ (say, $m<10$ ) density-wave patterns with different number of arms and wavelengths may coexist in the Galaxy 
(cf. Lin 1971; Bertin et al. 1977; Lin \& Lau 1979; Griv \& Wang 2014; Sellwood 2020). We may consider the multiple-armed spiral structure of the system in terms of several coexisting wave patterns.

(v) The sum $S$ increases and the coefficient of determination $\mathcal{R}^{2}$ decreases slightly with $m$ (cf. Griv et al. 2017, 2020, 2021). One can suggest that the dominant mode (that with lowest $S$ and highest $\mathcal{R}^{2}$ values) is therefore the one-armed $m=1$ wave. This conclusion must be regarded as unsettled until more extensive data are available. The latter is because the differences in $S$ and $\mathcal{R}^{2}$ of about 5 and 0.02 are not sufficiently large, smaller than about $5 \sigma$ errors for the differences.

(vi) Whatever $m \geq 1$ models we consider, the phase $\phi_{0}$ that we derived is $\approx 0^{\circ}$ and the local radial wavelength $\lambda_{0}$ is $\approx 1.5 \mathrm{kpc}$ for the minimum. Accordingly, the Sun is situated very close to a central, densest part of the spiral density-wave arm (very close to the potential well bottom of the spiral gravitational field). A $\lesssim$ $2 \mathrm{kpc}$ scale of inhomogeneity in the plane was already known from both the distribution of the young objects directly observable at large distances (Hou \& Han 2014; Reid et al. 2014; Nakanishi \& Sofue 2016; Reid et al. 2019; Hao et al. 2021) and the power spectrum of residual velocity fluctuations in the nearby disc (Bovy et al. 2015). To be consistent with the current ideas as to the Galactic spiral structure (Nakanishi \& Sofue 2016; Reid et al. 2019; Cantat-Gaudin et al. 2020; Chen et al. 2020; Khoperskov et al. 2020; Kounkel, Covey \& Stassun 2020; Poggio et al. 2021; Tarricq et al. 2021), we interpret this finding to mean that the kinematics of stars investigated reveals two segments of arms: the local Orion arm and the inner Sagittarius arm. This locates the Sun close to the arm centre (at the inner edge) of the Orion arm (cf. Griv et al. 2017, 2020, 2021). That, of course, is known from optical and radio observations (e.g. Hou \& Han 2014). The radial distance between the arms near the Sun, say, the Orion and Sagittarius ones, is $\approx 1.5 \mathrm{kpc}$. The centre of the Sagittarius arm is $\approx 1.5 \mathrm{kpc}$ from the Sun in the direction of the GC, and the width of the arms is $\approx 0.7 \mathrm{kpc}$.

(vii) The amplitude of the perturbing gravitational potential in the solar neighbourhood is

$\widetilde{\Phi} \sim \frac{\widetilde{v}_{r}^{2}+\widetilde{v}_{l}^{2}}{r_{0}^{2} \Omega_{0}^{2}} \Phi_{\text {basic }} \sim 0.03 \Phi_{\text {basic }}$,

that is, $\sim 3$ per cent of the background axisymmetric potential (cf. Minchev \& Famaey 2010; Siebert et al. 2012). Both the pitch angle of the pattern and the perturbing potential amplitude seem to be relatively small for all $m$-models considered. ${ }^{6}$ The fundamental LinShu approximation of tightly wound, small gravity disturbances used throughout the theory does not fall.

(viii) Two almost equal scales of spiral rarefaction-compression irregularity of the $v_{l}$ velocity field with the radial $\lambda_{0} \approx 1.5 \mathrm{kpc}$ and vertical $\xi_{0} \approx 1.0 \mathrm{kpc}$ wavelengths are estimated. The latter is the main new result. The result has been predicted by Griv \& Gedalin's (2012) modified theory of density waves. The value of $\xi_{0}$ is consistent with the theoretical expectation, that is, $\xi_{0} \lesssim \lambda_{0}$. Because young objects in the Galaxy are concentrated within dynamically cold thin disc with typical thickness of $h \lesssim 100 \mathrm{pc}$, in equations (2)-(7), the value of $|q z| \equiv\left|\left(2 \pi / \xi_{0}\right) z\right|$ is therefore small and we may assume it is zero in the lowest approximation to simplify the discussion. In practice, such accuracy is sufficient for the discussion of Lin-Shu-type modes of collective oscillations in the rotationally supported, dynamically cold systems.

\footnotetext{
${ }^{6}$ An astrophysicist might well consider a perturbation with $\left|\widetilde{\Phi} / \Phi_{\text {basic }}\right|$ of $<1 / 10$ to be quite small.
}

(ix) We found $\widetilde{v}_{r} \approx+6 \mathrm{~km} \mathrm{~s}^{-1}$ and $\widetilde{v}_{\varphi} \approx-36 \mathrm{~km} \mathrm{~s}^{-1}$ for the amplitudes of the streaming radial and tangential motions. Comparing table 1 with table 2 of Griv et al. (2020), we notice that $\widetilde{v}_{r}$ and $\widetilde{v}_{\varphi}$ derived from kinematics of OB stars at the level of $10-20 \mathrm{~km} \mathrm{~s}^{-1}$ are larger than the corresponding velocities derived from kinematics of main-sequence A-G stars. Such behaviour of streaming velocities is predicted by the theory. Indeed, the dynamical response of young OB population with the mean age of about $10^{7} \mathrm{yr}$ to the imposed oscillating potential was expected to be quite different from the response of older, on the average, $\mathrm{A}-\mathrm{G}$ population with the mean age of $10^{9} \mathrm{yr}$. The difference is regulated by the 'reduction factor' that takes into account the fact that the oscillating potential affects only weakly the objects of older population with high random velocities (Lin \& Shu 1966; Lin et al. 1969; Shu 1970; Griv et al. 2006).

(x) As for $\Omega_{0}$, its value is not too far from the estimates of the angular speed of the Sun derived from the Burns et al. (2014), Sharma et al. (2014), Kawata et al. (2019), Mróz et al. (2019), and Ablimit et al. (2020) samples of stars (as it should be because the waves are considered as small perturbations against an axisymmetric background).

(xi) Considering the $m \geq 1$ models, our sample of OB stars yields the first and second Oorts constants $A_{0} \approx+10 \mathrm{~km} \mathrm{~s}^{-1} \mathrm{kpc}^{-1}, B_{0}$ $\approx-19 \mathrm{~km} \mathrm{~s}^{-1} \mathrm{kpc}^{-1}$, whereas the conventional values are $A_{\text {conv }} \approx$ $+15 \mathrm{~km} \mathrm{~s}^{-1} \mathrm{kpc}^{-1}, B_{\text {conv }} \approx-12 \mathrm{~km} \mathrm{~s}^{-1} \mathrm{kpc}^{-1}$ (e.g. Bovy 2017; Wang et al. 2021). Accordingly, the Galaxy rotates more like a solid body and the mass density depends more weekly on $r$ (the local disc is more homogeneous) than in the conventional $m=0$ model (cf. Mishurov et al. 1979, p. 152). The value of $\mathrm{d} V_{\text {circ }} / \mathrm{d} r=$ $-A_{0}-B_{0}$ is positive, meaning that at our local neighbourhood, the rotational velocity $V_{\text {circ }}$ increases with distance from the GC. We find $V_{\text {circ }, 0} \equiv r_{0} \Omega_{0} \approx 241 \mathrm{~km} \mathrm{~s}^{-1}$. This value of the circular velocity is just slightly greater than the values $V_{\text {circ, } 0} \approx 233 \mathrm{~km} \mathrm{~s}^{-1}, V_{\text {circ, } 0} \approx$ $229 \mathrm{~km} \mathrm{~s}^{-1}, V_{\text {circ }, 0} \approx 234 \mathrm{~km} \mathrm{~s}^{-1}, V_{\text {circ }, 0} \approx 239 \mathrm{~km} \mathrm{~s}^{-1}$, and $V_{\text {circ }, 0}$ $\approx 235 \mathrm{~km} \mathrm{~s}^{-1}$ measured by McMillan (2017), Eilers et al. (2020), Mróz et al. (2019), VERA Collaboration (2020), and Nitschai et al. (2021), respectively, adopting the fundamental axisymmetric model.

(xii) The last point is that measurements of $u_{0} \approx-6 \mathrm{~km} \mathrm{~s}^{-1}$ (radially towards the GC) and $v_{0} \approx+10 \mathrm{~km} \mathrm{~s}^{-1}$ (in the direction of Galactic rotation) are not too far from the conventional values of $\approx-10 \mathrm{~km} \mathrm{~s}^{-1}$ and $\approx+15 \mathrm{~km} \mathrm{~s}^{-1}$ (Schönrich, Binney \& Dehnen 2010; Reid \& Brunthaler 2020; Gaia Collaboration 2021; Wang et al. 2021).

The construction of the rotation curve of the Galaxy within $2.5 \mathrm{kpc}$ of the Sun with the values of the parameters $\Omega_{0}$ and $\dot{\Omega}_{0}$ from Table 1 for the $m=4$ model is showed in Fig. 9. At the local solar neighbourhood, the rotational velocity $V_{\text {circ }}$ increases with distance from the GC. To repeat ourselves, in the LindbladOort approach distinct populations of objects with different random velocity dispersions (with different mean ages) have different average $V_{\text {circ }}$. Our rotation curve, in general, is in fair agreement with available previous studies (within the errors of them) by Mróz et al. (2019), Reid et al. (2019), and Bobylev \& Bajkova (2020).

\section{DISCUSSION}

In our own Milky Way Galaxy, a concentration of very young objects (young open clusters and Cepheids, OB associations, massive OB stars, HII regions, HI and giant molecular clouds) in the solar neighbourhood was already detected long ago in the inner Sagittarius and outer Perseus fragments of spiral arms, and the Orion fragment which is located between them. The Sun is spatially situated in the 


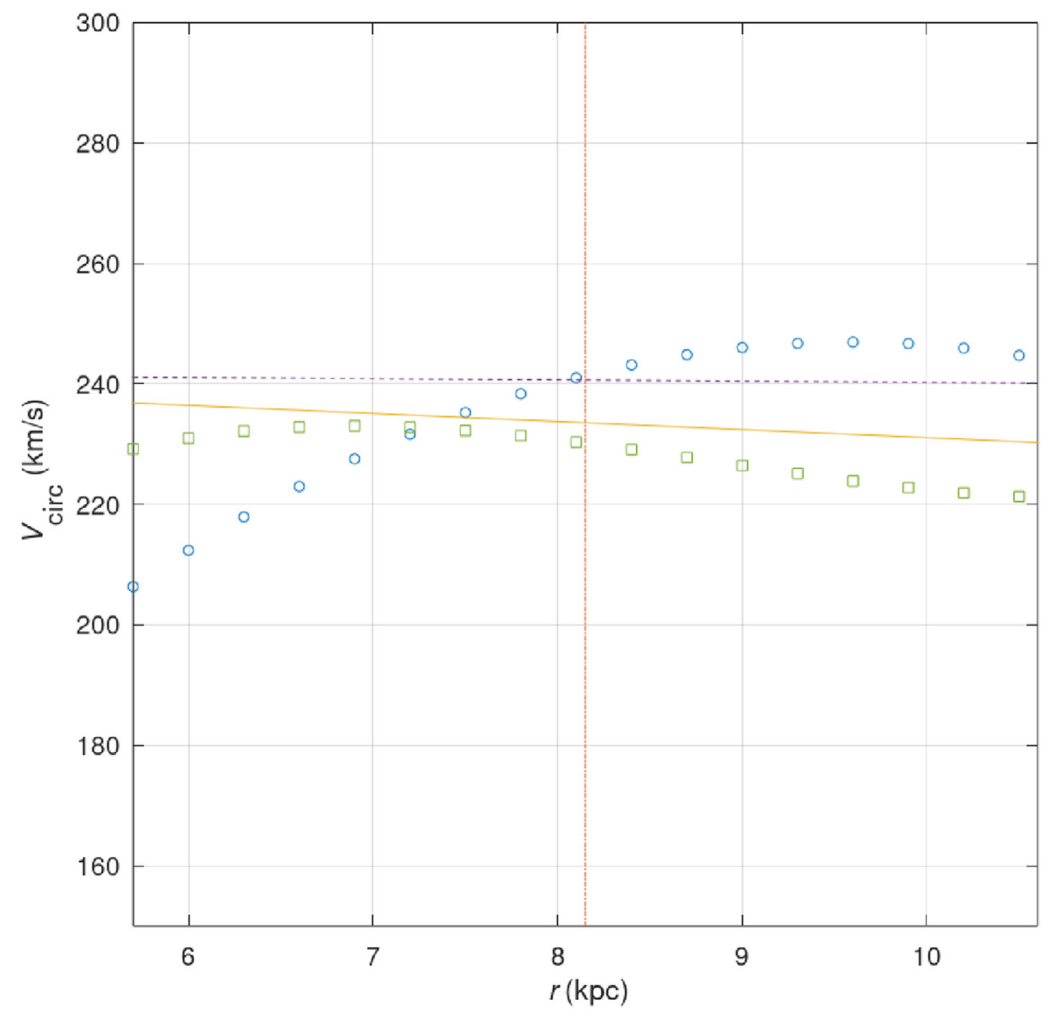

Figure 9. Rotation curve of the Galaxy for OB stars in the 2.5-kpc-scale solar vicinity for the $m=4$ model assuming $r_{0}=8.15 \mathrm{kpc}$ ( circles). The rotation curves obtained by Mróz et al. (2019) (a line), Reid et al. (2019) (a dashed line), and Bobylev \& Bajkova (2020) (squares) are also demonstrated. The vertical line marks the position of the Sun.

Orion fragment. As for this study, these spirals are nothing but LinShu-type unforced density waves, in which the self-gravitation of the fluctuations in density is taken into account (Fig. 1b). For these 'sausage' wave modes the vertical velocity of a mass element $v_{z}(+$ $z)=-v_{z}(-z)$ and, in particular $v_{z}(z=0)=0$, and matter oscillates in the direction of wave propagation.

We report and examine here the results of the dynamical analysis of a velocity field of stars on the assumption that the system is subject to moderately unstable, low-amplitude, tightly wound, and rigidly rotating density waves. The data of over $2600 \mathrm{O}$ and early B-type stars distributed along the Galactic disc in the region $d<$ $2.5 \mathrm{kpc}$ and $|z|<250 \mathrm{pc}$ are collected from the most updated Gaia EDR3 sample of spectroscopically confirmed young stars by $\mathrm{Xu}$ et al. (2021). Transverse along the longitude $v_{l}$ velocities of these objects are analysed by taking into additional account the effect of finite thickness of the disc. The data analysis made in the work appears to be sufficient to make strong conclusions about the spiral density-wave structure of the Galaxy. This is because the $S$ and $\mathcal{R}^{2}$ values returned from the analysis indicate that the $m$ $=1,2,3$, and 4 models are greatly more favourable than the $m$ $=0$ model without spiral arms. It is shown that there may exist two scales of spiral rarefaction-compression irregularity of the $v_{l}$ velocity field as already predicted by theory with the radial $\lambda_{0} \approx$ $1.5 \mathrm{kpc}$ and the vertical $\xi_{0} \approx 1.0 \mathrm{kpc}$ wavelengths. The latter is the main new and significant to be learnt from our numerical analysis. The amplitude of the perturbed potential in the solar neighbourhood under consideration $\widetilde{\Phi} \approx 0.03 \Phi_{\text {basic }}$ for all values of the number of spiral arms considered. In good agreement with the original Lin and Shu's suggestion, the tightly wound $\left(\lambda_{0} \ll r_{0}\right)$, spiral arms of the system therefore represent only small deviations of the potential from a potential distribution that is axisymmetric in the mean.

The phase of the wave at the Sun's location is $\phi_{0} \approx 0^{\circ}$. We argue respectively that the Sun is located very close to the Orion densitywave arm centre, between the nearby Sagittarius and Perseus arms. The centre of the Sagittarius arm is $\approx 1.5 \mathrm{kpc}$ from the Sun in the direction of the GC, and the width of the arms is $\approx 0.7 \mathrm{kpc}$. As follows, the Sun lies close to the centre, i.e. the densest part of the Orion feature (cf. Griv et al. 2017, 2020, 2021). This location of the Sun obtained in our model calculation is in excellent agreement with the observed space distribution of the standard arm tracers. ${ }^{7}$

The value of the vertical wavelength we have found is consistent with the theoretical prediction, namely $\xi_{0} \lesssim \lambda_{0}$ (see Griv \& Gedalin 2012). Considering the flat-component objects, in equations the terms which describe the $3 \mathrm{D}$ effects proportional to $|q z| \ll 1$, are thus small and we may omit it in the lowest approximation to simplify the discussion. For practical use, the usual 2D accuracy is sufficient for the discussion of Lin-Shu-type oscillation modes in the rotationally supported, dynamically cold systems of small thickness despite the presence of streaming $\propto \widetilde{v}_{z} \sin \phi \sin (q z)$ motion in the $z$-direction (see also Yuan \& Wallace 1973 and Soukup \& Yuan 1981 for a discussion).

\footnotetext{
${ }^{7}$ It is now possible to make a direct estimate of distances from the Sun to the spirals in the line of sight towards the GC. The strategy here is to compute their distances and the extinction using NIR photometry from the GALACTICNUCLEUS survey. By applying this method, Nogueras-Lara, Schödel \& Neumayer (2021) have also obtained an estimate of the distance to the Sagittarius arm of $\approx 1.6 \mathrm{kpc}$.
} 
In light of different $m$-models calculations completed in the last papers of a series (Griv et al. 2021; this work; see also Griv et al. 2017,2020 ) we think we are entitled to claim that the spiral pattern of the Galaxy is a superposition of moderately unstable $m$-armed independent waves. This might suggest a complicated multi-arm ('flocculent'?) spiral structure of the system (cf. the fluffy structure of the face-on Pinwheel galaxy, also known as M101). The Galaxy is probably not a pure grand design spiral, but a system showing not well-defined substructures. Xu et al. (2018), Chen et al. (2019), Hao et al. (2021), and Zari et al. (2021) have already claimed that the Galaxy is not a neat grand design spiral galaxy with well-organized arms.

The weighted least-square sum $S$ increases and the coefficient of determination $\mathcal{R}^{2}$ decreases slightly with $m$. The dominant mode (that with lowest $S$ and highest $\mathcal{R}^{2}$ values) is the $m=1$ wave for all four $m$-models considered. One may conclude that, even preliminary, our calculation favours a one-armed, azimuthally asymmetric dominant gravity perturbation in the solar neighbourhood. The $m$ $=1$ azimuthal asymmetry (or lopsidedness) is often seen in the distribution of stars and gas in disc-shaped galaxies especially in the outer parts of a system. About 30 per cent of galaxies in a magnitudelimited sample of 60 spiral galaxies show significant lopsidedness at large radii ( $r>1.5$ disc scale lengths) (Zaritsky \& Rix 1997). Spiral galaxies commonly display heavy morphological asymmetries in their H I gas (van Eymeren et al. 2011; Reynolds et al. 2020). The amplitudes of the $m=1,2,3$, and 4 modes in observations of the gas emission line [C II] of BRI 1335-0417, more than 12 Gyr old spiral galaxy in the distant Universe, have signal-to-noise ratios of 4.0, 6.0, 2.5 , and 2.4, respectively (Tsukui \& Iguchi 2021). An asymmetry between the locations of the arms on one side of the Galaxy compared with those on opposite side has already been revealed (Efremov 2011). Future works on the discrete oscillation modes near the Sun, especially on the most important $m=1$ mode, advocated in this work will benefit from the measurements of successive data releases of the Gaia astrometric mission.

The Gaia's DR2 line-of-sight (Griv et al. 2020) and EDR3 longitudinal (this work) velocities analyses performed in the last two papers of a series exhibit consistent findings.

Summarizing, the existence of spiral wave structure in the stellar $v_{l}$ velocity field is established. The velocity field of young stars in the solar vicinity is non-axisymmetric. Regular rarefactioncompression velocity oscillations occur both in the Galactic plane and in the direction perpendicular to the plane. The presence of such 3D structure gives direct support for the density-wave theory. Our calculation may suggest a complicated multi-arm spiral structure of the system. The Galaxy may belong to a general group of lopsided galaxies. The Sun lies close to the central, densest part of the Orion spiral wave feature. We also show that estimates obtained from an analysis of fully independent Gaia's $v_{\text {los }}$ and $v_{l}$ velocities are fairly consistent. This result based on recent observations creates further evidence to support the underlying Lin-Shu density-wave idea.

In closing, this study searches for the arm models only in the 2.5kpc-scale solar neighbourhood. Direct evidence of gravity unstable oscillation modes in a plane extending at least 10-12 kpc from the GC (e.g. Hachisuka et al. 2015; Sun et al. 2015) remains to be revealed.

\section{ACKNOWLEDGEMENTS}

This work has made use of data from the European Space Agency (ESA) mission Gaia (https://www.cosmos.esa.int/gaia), processed by the Gaia Data Processing and Analysis Consortium (DPAC; https: //www.cosmos.esa.int/web/gaia/dpac/consortium). Funding for the
DPAC has been provided by national institutions, in particular the institutions participating in the Gaia Multilateral Agreement. All of this work is based on the firm foundation of the study done by Chi Yuan in late 1960s and early 1970s. The authors are grateful to Ye Xu from the Purple Mountain Observatory for his generously providing sample of stars and important comments. EG would like to thank Irena Zlatopolsky for her useful technical backing and stimulation during the preparation of the paper. We express our gratitude to the anonymous referee for critical remarks, which helped to improve the paper. Financially, the efforts of EG for this work were supported in part by the Ministry of Immigrant Absorption, Israel in the framework of the program 'KAMEA.' Joint support from the Israel Science Foundation, the U.S-Israel Binational Science Foundation, and the Ministry of Science and Technology, Taiwan is acknowledged.

\section{DATA AVAILABILITY}

The data underlying this article were obtained from the VizieR database (Parallaxes and proper motions of OB stars from Gaia EDR3; Xu et al. 2021).

\section{REFERENCES}

Ablimit I., Zhao G., Flynn C., Bird S. A., 2020, ApJ, 895, L12

Antoja T. et al., 2018, Nature, 561, 360

Athanassoula E., Romero-Gómez M., Bosma A., Masdemont J. J., 2010, MNRAS, 407, 1433

Bennett M., Bovy J., 2019, MNRAS, 482, 1417

Bennett M., Bovy J., 2021, MNRAS, 503, 736

Bertin G., 1980, Phys. Rep., 61, 1

Bertin G., Lau Y. Y., Lin C. C., Mark J. W.-K., Sugiyama L., 1977, Proc. Natl. Acad. Sci. USA, 74, 4726

Bertin G., Lin C. C., Lowe S. A., Thurstans R. P., 1989, ApJ, 338, 78

Binney J., Tremaine S., 2008, Galactic Dynamics, 2nd edn. Princeton Univ. Press, Princeton, NJ

Bland-Hawthorn J., Gerhard O., 2016, ARA\&A, 54, 529

Bland-Hawthorn J. et al., 2019, MNRAS, 486, 1167

Bobylev V. V., Bajkova A. T., 2010, MNRAS, 408, 1788

Bobylev V. V., Bajkova A. T., 2014, MNRAS, 441, 142

Bobylev V. V., Bajkova A. T., 2015, MNRAS, 447, L50

Bobylev V. V., Bajkova A. T., 2020, Astron. Rep., 64, 1042

Bobylev V. V., Bajkova A. T., Stepanishchev A. S., 2008, Astron. Lett., 34, 515

Bobylev V. V., Bajkova A. T., Rastorguev R. S., Zabolotskikh M. V., 2021, MNRAS, 502, 4377

Bovy J., 2017, MNRAS, 468, L63

Bovy J., Bird J. C., Garcáa Pérez A. E., Majewski S. R., Nidever D. L., Zasowski G., 2015, ApJ, 800, 83

Burns R. A., Nagayama T., Handa T., Omodaka T., Nakagawa A., Nakanishi H., Hayashi M., Shizugami M., 2014, ApJ, 797, 39

Burton W. B., 1971, A\&A, 10, 76

Byl J., Ovenden M. W., 1978, ApJ, 225, 496

Cantat-Gaudin T. et al., 2020, A\&A, 640, A1

Carlin J. L. et al., 2013, ApJ, 777, L5

Castro-Ginard A. et al., 2021, A\&A, 652, 162

Chen B.-Q. et al., 2019, MNRAS, 487, 1400

Chen B.-Q. et al., 2020, MNRAS, 493, 351

Cheung E. et al., 2013, ApJ, 779, 162

Cohen J., 1988, Statistical Power Analysis for the Behavioural Sciences. Lawrence Erlbaum Associates, NJ

Crézé M., Mennessier M. O., 1973, A\&A, 27, 281

Ding P.-J., Xue X.-X., Yang C., Zhao G., Zhang L., Zhu Z., 2021, AJ, 162, 112

Efremov Yu. N., 2011, Astron. Rep., 55, 108

Eilers A.-C., Hogg D. W., Rix H.-W., Frankel N., Hunt J. A. S., Fouvry J.-B., Buck T., 2020, ApJ, 900, 186 
Feldman S. I., Lin C. C., 1973, SIAM Stud. Appl. Math., 52, 1

Fernández D., Figueras F., Torra J., 2001, A\&A, 372, 833

Gaia Collaboration, 2018, A\&A, 616, 11

Gaia Collaboration, 2021, A\&A, 649, 6

Goldreich P., Tremaine S., 1979, ApJ, 233, 857

Goldreich P., Tremaine S., 1980, ApJ, 241, 425

GRAVITY Collaboration, 2019, A\&A, 625, L10

Griv E., Gedalin M., 2012, MNRAS, 422, 600

Griv E., Wang H.-H., 2014, New Astron., 30, 8

Griv E., Gedalin M., Eichler D., Yuan C., 2000, Phys. Rev. Lett., 84, 4280

Griv E., Gedalin M., Yuan C., 2006, Adv. Space Res., 38, 47

Griv E., Jiang I.-G., Hou L.-G., 2017, MNRAS, 468, 3361

Griv E., Gedalin M., Shih I.-C., Hou L.-G., Jiang I.-G., 2020, MNRAS, 493, 2111

Griv E., Gedalin M., Jiang I.-G., 2019, MNRAS, 484, 218

Griv E., Gedalin M., Jiang I.-G., 2021, MNRAS, 503, 354

Grivnev E. M., 1981, SvA Lett., 7, 303

Hachisuka K., Choi Y. K., Reid M. J., Brunthaler A., Menten K. M., Sanna A., Dame T. M., 2015, ApJ, 800, 2

Hao C. J. et al., 2021, A\&A, 652, 102

Hou L.-G., Han J.-L., 2014, A\&A, 569, 125

Kawata D., Bovy J., Matsunaga N., Baba J., 2019, MNRAS, 482, 40

Khoperskov S., Gerhard O., Di Matteo P., Haywood M., Katz D., Khrapov S., Khoperskov A., Arnaboldi M., 2020, A\&A, 634, L8

Korchagin V. I., Marochnik L. S., 1975, SvA, 19, 8

Kounkel M., Covey K., Stassun K. G., 2020, ApJ, 160, 279

Lin C. C., 1971, Highlights Astron., 2, 88

Lin C. C., Lau Y. Y., 1979, SIAM Stud. Appl. Math., 60, 97

Lin C. C., Shu F. H., 1964, ApJ, 140, 646

Lin C. C., Shu F. H., 1966, Proc. Natl. Acad. Sci. USA, 55, 229

Lin C. C., Yuan C., Shu F. H., 1969, ApJ, 155, 721

Lin C. C., Yuan C., Roberts W. W., 1978, A\&A, 69, 181

Marochnik L. S., Suchkov A. A., 1981, Ap\&SS, 79, 337

McGaugh S. S., 2016, ApJ, 816, 42

McGaugh S. S., 2019, ApJ, 885, 87

McMIllan P. J., 2017, MNRAS, 94, 76

Mihalas D., Binney J., 1998, Galactic Astronomy, 2nd edn. Freeman, San Francisco

Minchev I., Famaey B., 2010, ApJ, 722, 112

Mishurov Yu. N., Pavlovskaya E. D., Suchkov A. A., 1979, SvA, 23, 147

Mishurov Yu. N., Zenina I. A., Dambis A. K., Melnik A. M., Rastorguev A. S., 1997, A\&A, 323, 775

Mróz P. et al., 2019, ApJ, 870, L10

Nakanishi H., Sofue Y., 2016, PASJ, 68, 5

Nitschai M. S., Eilers A.-C., Neumayer N., Cappellari M., Rix H.-W., 2021, ApJ, 916, 112
Nogueras-Lara F., Schödel R., Neumayer N., 2021, A\&A, 653, 33

Pavlovskaya E. D., Suchkov A. A., 1980, SvA, 24, 164

Poggio E. et al., 2021, A\&A, 651, 104

Reid M. J., Brunthaler A., 2020, ApJ, 892, 39

Reid M. J. et al., 2014, ApJ, 783, 130

Reid M. J. et al., 2019, ApJ, 885, 131

Reynolds T. N., Westmeier T., Staveley-Smith L., Chauhan G., Lagos C. D. P., 2020, MNRAS, 493, 5089

Rohlfs K., 1977, Lectures on Density Wave Theory. Lecture Notes in Physics 69. Springer, Berlin

Romero-Gómez M., Athanassoula E., Masdemont J. J., García-Gómez C., 2007, A\&A, 472, 63

Schönrich R., Dehnen W., 2018, MNRAS, 478, 3809

Schönrich R., Binney J., Dehnen W., 2010, MNRAS, 403, 1829

Sellwood J. A., 2020, MNRAS, 492, 3103

Sellwood J. A., Carlberg R. G., 2019, MNRAS, 489, 116

Sharma S. et al., 2014, ApJ, 793, 51

Shu F. H., 1970, ApJ, 160, 89

Siebert A. et al., 2012, MNRAS, 425, 2335

Skowron D. M. et al., 2019, Science, 365, 478

Soukup E., Yuan C., 1981, ApJ, 256, 376

Sun Y., Xu Y., Yang J., Li F.-C., Du X.-Y., Zhang S.-B., Zhou X., 2015, ApJ, 798, L27

Tarricq Y. et al., 2021, A\&A, 647, 19

Tsukui T., Iguchi S., 2021, Science, 372, 1201

van Eymeren J., Jütte E., Jog C. J., Stein Y., Dettmar R.-J., 2011, A\&A, 530, 30

VERA Collaboration, 2020, PASJ, 72, 50

Wang F., Zhang H.-W., Huang Y., Chen B.-Q., Wang H.-F., Wang C., 2021, MNRAS, 504, 199

Widrow L. M., Gardner S., Yanny B., Dodelson S., Chen H.-Y., 2012, ApJ, 750, L41

Williams M. E. K. et al., 2013, MNRAS, 436, 101

Xu Y. et al., 2018, A\&A, 616, L15

Xu Y., Hou L. G., Bian S. B., Hao C. J., Liu D. J., Li J. J., Li Y. J., 2021, A\&A, 645, L8

Yanny B., Gardner S., 2013, ApJ, 777, 91

Yuan C., 1969, ApJ, 158, 871

Yuan C., Wallace L., 1973, ApJ, 185, 453

Zari E., Rix H.-W., Frankel N., Xiang M., Poggio E., Drimmel R., Tkachenko A., 2021, A\&A, 650, 112

Zaritsky D., Rix H.-W., 1997, ApJ, 477, 118

This paper has been typeset from a $\mathrm{T}_{\mathrm{E}} \mathrm{X} / \mathrm{LT} \mathrm{E} \mathrm{X}$ file prepared by the author. 